I9I2 M.Z.Gr. Schätzungen Gr. Bb. Bem.

März $225^{\mathrm{h}} 45^{\mathrm{m}}$ T $5 y_{1}, \tau 6 x, N 0^{\mathrm{m}} \cdot 2>y 5^{\mathrm{m}} \cdot 34 \mathrm{G}$

$70 \pi 3.5 x, \times 7 y \quad 5.12 \mathrm{~B}$

$949 \pi 2.5 x \quad 4.90 \mathrm{~B}$

$23526 \tau 5 y, \times 6 y$

$642 \pi 4 y, \pi 2 x$

$5.08 \mathrm{~B}$

$850 \tau 2.5 z, \tau 663$ Aurigae $4.94 \mathrm{Bch}$

$849 \quad 3.5 y$

$95 \mathrm{I} \tau \mathrm{I} .5 x, x_{4} y \quad 4.84 \mathrm{~B}$

$24528 N$ O. I < $4.69 \mathrm{~B}$

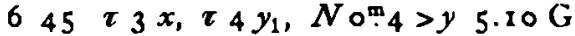

7 10 $x 5 \times$

$2556 N 0^{m} 5<\tau$

6 10 $N 0^{m} 2<\tau$

April 375 y $6 x$

$187 \circ b_{4} a$

741 b $5 a, b 2 c$

$1972 N^{\prime}=b$

836 b 10
$4.58 \mathrm{Bch}$

$5.09 \mathrm{~B}$ Mond

4.79 Bch Mond

5.70 ,

$6.43 \mathrm{~B}$

$6.44 \mathrm{~B}$

$6.18 \mathrm{~B}$

$6.24 B$ rötlich
Beobachter: $\mathrm{G}=M$. Gratschew, Observator; $B=$ $W$. Baranow, Assistent; $\mathrm{Bch}=T$. Banachicwicz, Assistent. $B$ und $G$ benutzten ein gewöhnliches Opernglas, Bch ein Trieder-Binokel Zeiß (Vergr. 6).

$$
\begin{array}{rr} 
& \begin{array}{r}
\text { Vergleichsterne. } \\
\mathrm{BD}+29^{\circ} 1154
\end{array} \\
+281168 & 4055=49 \text { Auriga } \\
+291293 & 409 \mathrm{I}=53 \text { Auriga } \\
+321414 & 4200 \\
+341481 & 4229=9 \text { Genino } \\
+321433 & 4243 \\
+331433 & 4271 \\
+321460 & 4317 \\
+291441 & 4337= \\
+301439 & 4401=x \text { Gemino } \\
+391882 & 4402=63 \text { Auriga } \\
+391690 & 4084=51 \text { Auriga } \\
\text { Kasan, 1913 Juli } 3 .
\end{array}
$$

\title{
L'astrophotomètre pour mesurer l'intensité des surfaces et les observations photométriques sur la Lumière Zodiacale. Par B. Fessenkoff.
}

L'astrophotomètre pour mesurer l'intensité des surfaces qui a été construit par M. Mouronval, mécanicien de l'observatoire de Paris, suivant mes indications, a la construction suivante. Un petit réfracteur possède, normalement à son axe, un tube qui est placé pas loin de l'oculaire. Ce tube consiste en deux tubes secondaires qui sont joints avec des vis. Au bout du second se place une petite lampe électrique de 8 volts qui fonctionne dans le photomètre comme source de lumière artificielle. Cette lampe reçoit l'énergie d'une batterie d'accumulateurs. Entre les deux tubes se trouve un micromètre, qui change la quantité de lumière sortant de la lampe. Il consiste en deux diaphragmes sectoriaux a quatre bouts. L'angle de chaque secteur est de $45^{\circ}$. Entre ces secteurs se trouvent les aires sectoriates ouvertes du même angle. Un diaphragme est fixé, l'autre se déplace devant lui à l'aide d'une vis micrométrique. Tout près du micromètre se troure la lentille biconvexe; puis au bout du premier tube lié directement avec le réfracteur, se trouve un petit diaphragme qui a un diamètre à peu près d'un millimètre; il est couvert d'un verre mat. La longueur des tubes est arrangée de telle façon qu'un focus de la lentille soit dans la lampe electrique et l'autre sur la surface du verre nat. Du côté inverse de ce diaphragme qui est tourné vers le refracteur se trouve la lentille plan-convexe. Elle sert à éviter la diffusion superflue de la lumière. A l'intérieur de la lunette est fixé un petit miroir. Ensuite dans le plan focal de l'objectif est placé un prisme à angle de réfraction assez aigu; l'arête du prisme passe par le centre du champ de la vision. Les rayons de lumière qui sortent du diaphragme au verre mat tombent sur le prisme après s'être réfléchis sur le miroir. Ce miroir doit être orienté de telle façon que les rayons après être réfractés dans le prisme entrent dans l'oculaire parallélement à l'axe optique de l'instrument. Il est évident que les rayons réfléchis entrant dans le tube de l'oculaire a travers la moitie du champ de la vision, qui n'est pas occupée par le prisme, conservent leur direction inclinée sur l'axe optique de l'instrument et restent invisibles pour l'observateur. Dans ce champ de la vision l'observateur examine à l'aide d'un oculaire l'image de l'objet à observer. Certes, les rayons de cet objet d'observation passent non seulement par l'aire libre du champ de vue, mais aussi par le prisme; mais après s'être réfractés dans sa substance ils n'atteignent pas l'œil de l'observateur et demeurent invisibles. Nous voyons que ce prisme sert à partager la lumière artificielle de comparaison et la lumière provenant de l'objet à examiner. Dans ces conditions une moitié du champ de vue est occupée par la lumière qui sort de l'objectif et l'autre par la lumière de la lampe électrique. Les observations consistent déterminer la lecture du micromètre quand les deux moitiés se confondent en un cercle également éclairé. Je laisse au côté les détails secondaires qui sont introduites pour faciliter les observations selon la nature de l'objet à examiner, de même que l'examen de l'instrument. Je vais exposer les résultats de mes observations sur la Lumière Zodiacale que j'ai faites aux mois de février et de mars 1913 à l'observatoire de Meudon et aux mois d'avril et de mai à l'observatoire de Nice, grâce à l'aimable autorisation de M. Deslandres et de $M$, le général Bassot. Les éléments de mes observations photométriques étaient: lectures du micromètre, du temps et de la distance zénithale jointes au pointement sur une carte du ciel des parties ouservées de la Lunière Zodiacale. Pendant toute la durée des observations l'instrument était maintenu à la même hauteur, pour que la correction provenant de l'absorption atmosphérique, qui est connue assez imparfaitement, soit sensiblement la même. Mes résultats peuvent être représentés sous la forme d'une table moyenne pour Nice et Meudon, dont tous les chiffres sont déjà affranchis de l'absorption atmosphérique. Dans la forme réduite elle est la suivante: 


\begin{tabular}{c||c|c|c|c|c|c}
\hline \multicolumn{1}{c||}{$s$} & $0^{\circ}$ & $4^{\circ}$ & $8^{\circ}$ & $12^{\circ}$ & $16^{\circ}$ & $20^{\circ}$ \\
\hline $34^{\circ}$ & 27.5 & 24.9 & 19.4 & 15.4 & 14.0 & 13.8 \\
38 & 2.3 .8 & 21.6 & 17.2 & 14.1 & 13.0 & 12.9 \\
42 & 20.6 & 18.9 & 15.4 & 13.0 & 12.3 & 12.2 \\
46 & 17.9 & 16.5 & 13.9 & 12.1 & 11.5 & 11.5 \\
50 & 15.6 & 14.6 & 12.6 & 11.3 & 10.9 & 10.9 \\
54 & 13.8 & 13.0 & 11.6 & 10.7 & 10.4 & 10.4 \\
$5^{8}$ & 12.4 & 11.9 & 10.8 & 10.2 & 10.0 & 10.0
\end{tabular}

$l$ est la distance angulaire du Soleil et $s$ la distance de l'axe de la Lumiere Zodiacale. Si cet axe coïncide avec l'écliptique, nous avons que: $l=\lambda-\odot$ et $s=\beta$ avec les notations usuelles. Les chiffres de cette table peuvent être représentés par la formule: $i=k+n(\cos 45)^{b}$ entre les limites $-20^{\circ} \leqq s \leqq+20^{\circ}, 34^{\circ} \leqq l \leqq 60^{\circ}$

où

$$
\begin{aligned}
& k=13.8-0.08 \operatorname{arctg}\left[0.045\left(l-34^{\circ}\right)\right] \\
& n=13.7-0.2 \operatorname{arctg}\left[0.0625\left(l-34^{\circ}\right)\right] \\
& b=6.6 \sin \left(l+20^{\circ}\right) .
\end{aligned}
$$

En appliquant mes résultats à l'examen des théories émises sur la nature de la Lumière Zodiacale j'ai trouvé qu'ils peuvent être représentés dans la seule supposition que la Lumière Zodiacale est un amas de poussière cosmique entourant le Soleil. Dans son plan équatorial la densité doit varier en raison inverse de la distance du Soleil.

Paris, I 9 I 3 Juillet 31 .

\section{B. Fessenkoff.}

\section{Sur la théorie des queues des comètes.}

Dans les Publications de l'Observatoire de Jurjew (Dorpat) j'ai écrit en russe une note sur les queues des comètes. Pour éviter des malentendus possibles (vgl. Kopff. Über die Schweifstrahlen, A. N. 4640 p. 143) je suis obligé de donner ici un résumé de cette note en langue plus répandue que la nôtre.

Je fais quelques remarques sur la queue de la comète I907 IV (Daniel); je montre que la forme rectiligne de ses rayons n'est pas en contradiction avec la théorie de Bredichine. C'est ce que M. Kopff a remarqué d'ailleurs lui même.

Soit $\mu$ la constante de la force centrale, $g$ et $G$ la valeur et l'angle de la direction de la vitesse initiale de la particule dans le plan de son mouvement. Le problème qui se pose est le suivant: d'après la forme de la queue il faut trouver trois constantes $\mu, g$ et $G$, qui peuvent être d'ailleurs quelconques.

Soient $\xi$ et $\eta$ les coordonées relatives d'une particule. En intégrant les équations différentielles du mouvement par des séries, on trouve $\xi$ et $\eta$ en fonction du temps $t$ :

Si $a_{1} \neq 0$, on a

$$
\begin{aligned}
& \xi=a_{1} t+a_{2} t^{2}+a_{3} t^{3}+\cdots \\
& \eta=b_{1} t+b_{2} t^{2}+b_{3} t^{3}+\cdots .
\end{aligned}
$$

$$
t=\alpha_{1} \xi+\alpha_{2} \xi^{2}+\alpha_{3} \xi^{3}+\cdots
$$$$
\text { d'où } \quad \eta=\beta_{1} \xi+\beta_{2} \xi^{2}+\beta_{3} \xi^{3}+\cdots \text {. }
$$

Toutes les constantes $a, b, \alpha$ et $\beta$ sont ici des fonctions de $\mu, g$ et $G$.
Pour que les séries écrites plus haut soient admissibles il faut que $t$ soit suffisamment petit; mais d'autre part, si $t$ est tellement petit que la série (I) n'a qu'un terme sensible en $\xi$, c'est à dire si l'on peut poser:

$$
\eta=\beta_{1} \xi
$$

on n'en peut tirer que quelques conclusions sur la direction de la vitesse initiale. Les trajectoires des particules dans les limites adoptées sont des lignes droites, c'est ce qu'on voit, par exemple, sur les dessins de M. Kopff pour la comète r 907 IV (Daniel) (Publikationen des Astrophys. Instituts Heidelberg, Bd. III Nr. 7). Ces dessins ne sont donc pas en contradiction avec la théorie de Bredichine.

Supposons maintenant, que la queue est prise tellement longue, que la série ( $r$ ) contienne trois termes sensibles en $\xi$. Je dis que même dans ce cas les données des observations sont insuffisantes pour qu'on en puisse faire des conclusions sur la theorie de Bredichine. Si l'on a trouvé $\mu, g$ et $G$ d'après les trois coefficients $\beta_{1}, \beta_{2}, \beta_{3}$, ce ne sera qu'une interpolation pure. Il faut encore que le calcul des points plus éloignés de la queue soit en accord avec l'observation. Pour ce calcul Bredichine a donné les formules exactes. Récemment M. Pokrowsky en suivant les méthodes de Bredichine a représenté par la théorie en question toute la queue de la comète r 9 ro I (Publications de l'Observatoire de Jurjew T. 2 (.4).

Odessa, Alexander-Park, I 9 I 3 Juillet.

Aufforderung. Herr Observator F. Van der Bilt, Universitäts-Sternwarte Utrecht (Holland) hat eine Bearbeitung des Lichtwechsels von R Sagittae, V Vulpeculae und RV Tauri in Angriff genommen, und bittet daher, unpublizierte oder an schwer erreichbaren Stellen veröffentlichte Originalschätzungen dieser Sterne einsenden zu wollen.

Benennung. Der Planet $744\left[\mathrm{Ig}_{3} \mathrm{QW}\right]$ hat vom Entdecker F. Rheden den Namen Aguntina erhalten.

Berichtigung zu Berliner Jahrbuch 1915 p. (24) und (73) bei 459 Signe Größe statt : $14^{\mathrm{m}} \cdot 9$ lies : $12^{\mathrm{m}} \cdot 4$. zu Nr. 4685 Bd. 196 p. 78 Tabelle $B_{2}$ für 1901.88 statt: -29 lies: +49 .

$4688 "$ " 133 Planet 7441913 März 14 Rom $\Delta \alpha+13^{\text {s. }} 4$ I $\Delta 8+1.2$ zu streichen, vergl. A. N. $4645,4683$. 4689 "145 vorletzte Zeile des 2. Absatzes statt: Stakke lies: Skakke.

Inhalt zu Nr. 4693. G. Abetti. Misure di Stelle Doppie. 217. - Beobachtungen des Kometen 19i3a (Schaumasse). 223. - Wl. Srebriansky Beobachtung der Perseiden 1904. 227. - D. Dubiago. Helligkeitsschätzungen der Nova Geminorum 2 auf der EngelhardtSterniwarte. 227. - B. Fessenkoff. L'astrophotomètre pour mesurer l'intensité des surfaces et les observations photométriques sur la Lumière Zodiacale. 229. - $A$. Orloff. Sur la thérie des queues des comètes. 23I. - Kleine Mitteilungen. 231 . Berichtigungen. 231. 